\title{
Effect of early calf-hood nutrition on the transcriptomic profile of subcutaneous adipose tissue in Holstein-Friesian bulls
}

\author{
Anne-Marie English ${ }^{1,2} \mathbb{D}$, Sineád M. Waters ${ }^{1}$, Paul Cormican ${ }^{1}$, Colin J. Byrne ${ }^{1,3}$, Seán Fair ${ }^{2}$ and David A. Kenny ${ }^{1,3^{*}}$
}

\begin{abstract}
Background: Adipose tissue is a major endocrine organ and is thought to play a central role in the metabolic control of reproductive function in cattle. Plane of nutrition during early life has been shown to influence the timing of puberty in both male and female cattle, though the exact biological mechanisms involved are currently unknown. The aim of this study was to investigate the effect of early calf-hood nutrition on the transcriptomic profile of subcutaneous adipose tissue in Holstein-Friesian bulls to identify possible downstream effects on reproductive physiology.

Results: Holstein-Friesian bull calves with a mean $( \pm$ S.D.) age and bodyweight of $19( \pm 8.2)$ days and $47.5( \pm 5.3) \mathrm{kg}$, respectively, were assigned to either a high $(n=10)$ or low $(n=10)$ plane of nutrition. Calves were fed in order to achieve an overall growth rate of 1.08 and $0.57 \mathrm{~kg} /$ day for the high and low plane of nutrition treatments, respectively. At 126 days of age, the bulls were euthanized, subcutaneous adipose tissue samples were harvested and RNAseq analysis was performed. There were 674 genes differentially expressed in adipose tissue of calves on the low compared with the high plane of nutrition $(P<0.05$; FDR $<0.05$; fold change $>2.0$ ). High plane of nutrition positively altered the expression of genes across an array of putative biological processes but the most dominant cellular processes affected were cellular energy production and branched chain amino acid degradation. A high plane of nutrition caused upregulation of genes such as leptin (LEP) and adiponectin (ADIPOQ), which are known to directly affect reproductive function.
\end{abstract}

Conclusions: These results provide an insight into the effect of augmenting the plane of nutrition of Holstein-Friesian bull calves in the prepubertal period on the transcriptome of adipose tissue.

Keywords: Gene expression, Reproduction, Adipogenesis, Amino acids, Mitochondrial dysfunction, Puberty

\section{Background}

The advent of genomic selection in the dairy industry has resulted in the identification of sires for use in artificial insemination within weeks of birth and semen from these young bulls is in high demand once they reach puberty. Studies have shown that plane of nutrition in early calf-hood plays a critical role in the timing of the onset of puberty in bulls [1-3]. Recently, our research group has demonstrated that a high plane of nutrition prior to, but not after 6 months of age can accelerate

\footnotetext{
* Correspondence: david.kenny@teagasc.ie

${ }^{1}$ Animal and Bioscience Research Department, Teagasc Grange, Dunsany, Co. Meath C15 PW93, Ireland

${ }^{3}$ School of Agriculture and Food Science, University College Dublin, Belfield,

Dublin 4, Dublin D04 N2E5, Ireland

Full list of author information is available at the end of the article
}

puberty in Holstein-Friesian bulls by approximately 1 month [3]. This has been associated with the advancement of a transient rise in systemic concentrations of luteinising hormone (LH), which normally occurs between approximately 8 and 20 weeks of age [4]. There are many organs implicated in signalling nutritional status to the hypothalamus and anterior pituitary to stimulate gonadotropin releasing hormone ( $\mathrm{GnRH})$ and $\mathrm{LH}$ secretion, respectively, with white adipose tissue (WAT) key amongst these.

White adipose tissue has traditionally been known for its role in energy storage and release when energy expenditure is greater than energy intake; however, WAT is metabolically active and can therefore influence many bodily systems. The function of WAT in metabolic and reproductive processes is complex but it has been postulated

(c) The Author(s). 2018 Open Access This article is distributed under the terms of the Creative Commons Attribution 4.0 International License (http://creativecommons.org/licenses/by/4.0/), which permits unrestricted use, distribution, and reproduction in any medium, provided you give appropriate credit to the original author(s) and the source, provide a link to the Creative Commons license, and indicate if changes were made. The Creative Commons Public Domain Dedication waiver (http://creativecommons.org/publicdomain/zero/1.0/) applies to the data made available in this article, unless otherwise stated. 
that there is cross-talk between adipokines and the hypothalamic-pituitary-testicular (HPT) axis. Adipogenesis and fat development are influenced by genetics, diet, body depot location and age $[5,6]$. There is evidence that offering calves high starch diet during early calfhood induces precocious adipogenesis and lipid filling [7]. It is postulated that the period of potential for stem cells to differentiate into adipocytes is very limited during early life development [8]. Adipose hormones such as leptin production increases proportionally in line with an increase in body fat [9]; evidently then, it is a signal of energy sufficiency [10]. It has been reported that subcutaneous adipose tissue has a higher concentration of both leptin and adiponectin protein than that of visceral adipose [11]. Leptin signals various bodily systems, including the HPT axis, of metabolic energy status and reserves [12]. Studies have shown that while there is an absence of leptin receptors on some key reproductively related cells such as GnRH neurons [13]; the effects of leptin on GnRH release have been shown to be mediated by kisspeptin in some species [14]. Adiponectin, another adipokine hormone, has been shown to have its receptors, ADIPOR1 and ADIPOR2, expressed in the anterior pituitary and the arcuate nucleus in the hypothalamus of cows [15]; therefore, adiponectin employs two potential pathways to signal to the HPT axis.

It has been reported in children that consistent excessive energy intake during early life development can contribute to precocious puberty [16]. In bull calves of dairy breeds early pubertal onset is advantageous to facilitate early semen collection. The exact metabolic mechanisms are unknown; hence this study aimed to examine the effect of early life plane of nutrition on global gene expression profiles of adipose tissue in Holstein-Friesian bulls within the particular context of potential latent implications for HPT function. Much of the published work to-date on this topic has focused on the effect of BCS (body condition score) on adipose tissue hormone signalling in post pubertal animals and there is a dearth of information on the role of adipose tissue in stimulating the HPT axis and its potential as a key regulator of the timing of puberty onset in cattle.

\section{Methods}

All procedures involving animals were approved by the Teagasc Animal Ethics Committee (TAEC30/2013) and were licensed by the Health Products Regulatory Authority, Ireland in accordance with the European Union Directive 2010/36/EU.

\section{Animal model}

This experiment was conducted as part of a larger study designed to examine the effect of early calf-hood nutrition on the physiological control of the HPT axis. HolsteinFriesian bull calves $(n=20)$ with a mean $( \pm$ S.D. $)$ age and bodyweight of $19( \pm 8.2)$ days and $47.5( \pm 5.3) \mathrm{kg}$, were purchased from commercial dairy farms and blocked based on sire, initial weight, farm of origin and age and assigned to either a high $(n=10)$ or low $(n=10)$ plane of nutrition. Calves were individually fed milk replacer and concentrates using an electronic feeding system (Forster-Tecknik Vario, Engen, Germany). The chemical analysis of the milk replacer and the concentrate offered during the trial is outlined in Tables 1 and 2. After 5 days of acclimatisation, the high treatment were offered $1200 \mathrm{~g}$ of milk replacer in $8 \mathrm{~L}$ of water daily, together with concentrate ad libitum. Animals in the low treatment group were allocated $500 \mathrm{~g}$ of milk replacer in $4 \mathrm{~L}$ of water and a further allocation of a maximum of $1 \mathrm{~kg}$ of concentrate daily. All calves were weaned when consuming a minimum of $1 \mathrm{~kg}$ of concentrate for 3 consecutive days, at a mean age $( \pm$ S.D. $)$ of $82( \pm 3.9)$ days. Following weaning, the high treatment group was offered ad libitum concentrates, while the low treatment group received $1 \mathrm{~kg}$ of concentrate daily. All calves had individual daily access to approximately $0.5 \mathrm{~kg}$ of straw as well as ad libitum access to fresh water throughout the trial period. Animals were weighed weekly over 16 weeks of the trial.

\section{Tissue collection}

The calves were euthanized at a mean age $( \pm$ S.D.) of 126 $( \pm 1.1)$ days, using an intravenous overdose of sodium pentobarbitone. Death was confirmed by lack of ocular response. Subcutaneous adipose samples were obtained from the flank of the carcass following slaughter. The epidermis and dermis layers were cut to reveal the subcutaneous adipose. The tissue samples were washed with Dulbecco's PBS and any blood vessels were removed. The tissue was subsampled and the subsamples were either (i) snap-frozen in liquid nitrogen and subsequently stored at $-80{ }^{\circ} \mathrm{C}$ for long term storage pending further analysis or (ii) fixed in $10 \%$ neutral buffered formalin (Sigma-Aldrich, Wicklow, Ireland) and embedded in paraffin in accordance to the standard procedures. Sections $(5 \mu \mathrm{m}$ thick) were dehydrated in ascending concentrations grades of alcohol, followed by clearing with xylene and were stained for gross anatomy using haematoxylin and eosin (H\&E) stain.

Table 1 Chemical composition of milk replacer

Milk Replacer

\begin{tabular}{ll}
\hline Chemical composition $(\mathrm{g} / \mathrm{kg})$ & \\
ADF & $12.0 \pm 1.98$ \\
Crude ash & $65.7 \pm 2.22$ \\
CP & $216.3 \pm 1.24$ \\
DM (\%) & $96.7 \pm 0.15$ \\
NDF & $5.1 \pm 1.00$ \\
Oil B & $235.0 \pm 44.10$ \\
\hline
\end{tabular}

$A D F$ Acid Detergent Fibre, $C P$ Crude Protein, DM Dry matter, NDF Neutral Detergent Fibre, Oil B Acid Hydrolysis 
Table 2 Diet and chemical composition of concentrate diet offered

\begin{tabular}{|c|c|}
\hline & Concentrate \\
\hline \multicolumn{2}{|l|}{ Diet composition (\%) } \\
\hline Rolled Barley & 26.5 \\
\hline Soya bean meal & 25 \\
\hline Maize & 15 \\
\hline Beet pulp & 12.5 \\
\hline Soya hulls & 12.5 \\
\hline Molasses & 5 \\
\hline Mineral and vitamins & $2.5^{\mathrm{a}}$ \\
\hline Vegetable oil & 1 \\
\hline \multicolumn{2}{|c|}{ Chemical composition (g/kg) } \\
\hline ADF & $103.1 \pm 6.76$ \\
\hline Crude ash & $68.8 \pm 0.91$ \\
\hline $\mathrm{CP}$ & $167.9 \pm 1.86$ \\
\hline DM (\%) & $88.9 \pm 0.66$ \\
\hline NDF & $204.3 \pm 18.2$ \\
\hline Oil B ${ }^{b}$ & $30.8 \pm 0.72$ \\
\hline
\end{tabular}

\section{Adipocyte cell number and diameter}

Adipocyte cell number and cell size were analysed using Aperio ImageScope (v12; Leica Biosystems, Wetzlar, Germany) from the H\&E stain slides. Five square sections $\left(0.25 \mathrm{~mm}^{2}\right)$ were electronically generated and randomly assigned on each slide and every adipocyte within these square sections was counted manually. The diameter of 200 adipocytes per animal, selected at random, within these square sections was measured at the widest point for each cell, using the ruler tool from the ImageScope software. The evaluator was blind to the treatments.

\section{Statistical analysis on adipocyte cell size and number}

Data for adipocyte cell size and number and bodyweight were analysed using the procedures of Statistical Analysis Software (SAS version 9.3, Cary, NC, USA) and were tested for normality (UNIVARIATE procedure). Residuals for this data were found to be normally distributed and were statistically analysed using ANOVA (MIXED procedure). Animal was the experimental unit and was included as a random effect. Sampling time (week of weight recording) was included in the statistical models as a repeated measure for weights. All results are presented as mean \pm s.e.m.

\section{RNA isolation and purification}

Total RNA was extracted using RNeasy Lipid Tissue Mini kit from $100 \mathrm{mg}$ of subcutaneous adipose (Qiagen, Manchester, UK). RNA was purified using the RNA Clean \& Concentrator kit (Zymo Research, Irvine, CA,
USA). The quantity of the RNA isolated was determined by measuring the absorbance at $260 \mathrm{~nm}$ using a Nanodrop spectrophotometer ND-1000 (Nanodrop Technologies, Wilmington, DE, USA). RNA quality was assessed on the Agilent Bioanalyzer 2100 (Agilent Technologies Ireland Ltd., Dublin, Ireland) using the RNA 6000 Nano Lab Chip kit (Agilent Technologies Ireland Ltd., Dublin, Ireland). Samples had an RNA Integrity Number (RIN) of mean $( \pm \mathrm{SD})$ of $7.6( \pm 0.77)$.

\section{RNAseq library preparation and sequencing}

Twenty cDNA libraries were prepared from high quality RNA using an Illumina TruSeq RNA Sample Preparation kit v2 following the manufacturer's instructions (Illumina, San Diego, CA, USA). For each sample, $1 \mu \mathrm{g}$ of total RNA was used for CDNA preparation. All libraries were validated on the Agilent Bioanalyzer 2100 using the DNA 1000 Nano Lab Chip kit (Agilent Technologies Ireland Ltd., Dublin, Ireland). Individual RNAseq libraries were pooled (10 libraries per lane) based on their respective sample-specific$6 \mathrm{bp}$ adaptors and sequenced at $100 \mathrm{bp} /$ sequence single-end reads using an Illumina HiSeq 2500 sequencer.

\section{RNAseq data analyses}

Raw sequence reads were checked for quality using FASTQC software (version 0.10.0) (http://www.bioinformatics.babraham.ac.uk/projects/fastqc/). Input reads were then aligned to the bovine reference genome (UMD3.1) using Spliced Transcripts Alignment to a Reference (STAR) aligner. Reads were only aligned if they contained fewer than two mismatches with the reference genome and uniquely mapped to the reference genome. The software package HTSeq (v0.5.4p5) was used to calculate the number of sequence reads overlapping all protein coding genes from the ENSEMBLv74 annotation of the bovine genome. The number of read counts mapping to each annotated gene from HTSeq was then collated into a single file and used for calculation of subsequent differential gene expression. Only uniquely mapped reads were used for subsequent differential gene expression analysis.. Reads were filtered out if they contained fewer than five counts per million in nine samples out of the total sample number from subsequent analysis. The R (v 3.01) Bioconductor package EdgeR (v3.2.4), was applied to identify statistically significant differentially expressed genes (DEGs).

\section{Pathway analysis}

Biological pathways were identified using GOSeq software (v1.14.0) [17] and Kyoto Encyclopaedia of Genes and Genomes (KEGG) pathway annotations [18] were used to identify biological pathways that were over-expressed among DEGs. GOSeq is an application for executing gene ontology analysis on RNAseq data while accounting for biases [17]. The online tool BioMart (www.ensembl.org/ 
biomart/martview) was used to convert the bovine gene IDs, extracted from GOSeq, into human orthologs. These human orthologs were inputted into KEGG (http://www. genome.jp/kegg/pathway.html). To examine the molecular functions and biological pathways, the RNAseq data was also analysed using Ingenuity Pathway Analysis (IPA) (Ingenuity Systems, Redwood City, CA; https:// www.qiagenbioinformatics.com/products/ingenuitypathway-analysis/).

\section{Results}

\section{Animal performance and adipose tissue histology}

Average daily bodyweight gain from the start to the end of the study was $1.08 \pm 0.03 \mathrm{~kg}$ and $0.57 \pm 0.03 \mathrm{~kg}$ for high and low plane of nutrition groups, respectively and this translated into a bodyweight difference of $53.8 \mathrm{~kg}$ at slaughter between the bulls of the high compared to the low treatment group $(160.9 \pm 3.98 \mathrm{~kg}$ versus $107.1 \pm 3.19 \mathrm{~kg}$, respectively; $P<0.001)$. Adipocyte cell number $(291 \pm$ 47.2 cells $/ 1.25 \mathrm{~mm}^{2}$ versus $7.5 \pm 4.0$ cells $/ 1.25 \mathrm{~mm}^{2}, P<$ 0.001 ; Fig. 1$)$ and cell diameter $(36.9 \pm 2.89 \mu \mathrm{m}$ versus $3.7 \pm 1.94 \mu \mathrm{m}, P<0.001$ ) were both greater in the high compared with the low plane of nutrition animals, respectively.

\section{Differential gene expression}

The mean \pm SD number of raw reads per sample was; $18,670,029 \pm 4,093,759$. Approximately $89 \%$ of the reads were uniquely mapped to the reference bovine genome after alignment. A multi-dimensional scaling (MDS) plot was created in Edge- $R$ which estimated the degree of similarity between samples from the high compared to the low nutritional treatments. The MDS plot showed a separation between the two treatments (Fig. 2). There were 745 differentially expressed genes (DEG) between the two nutritional treatments $(P<0.05$; False Discovery Rate $<0.05$; fold change $>2.0$ ). One hundred and sixty four genes had increased expression, while 581 had decreased expression in the low plane of nutrition compared to the high plane of nutrition. The RNAseq unprocessed data have been deposited in NCBI's Gene Expression Omnibus and area accessible via GEO series accession number GSE97674.

\section{Pathway analysis}

There were 678 DEG from the original 745 that mapped successfully to a molecular/biological pathway using IPA and these DEG were analysed and allocated to a biological function within IPA. Information on the effect of nutritional treatment on the molecular and cellular functions and on the biochemical pathways affected are presented in Additional files 1 and 2, respectively. Results of KEGG pathway analysis using GOSeq (FDR $<0.05)$ indicated that 23 KEGG pathways were over-represented among DEG in the low treatment compared to the high treatment at 18 weeks of age (Table 3). These enriched pathways included metabolic pathways, valine, leucine and isoleucine degradation and citrate cycle. After analysis using IPA, the following pathways and processes were identified as the most influenced by the differential nutritional regimen at the level of subcutaneous adipose tissue: energy production via mitochondrial dysfunction $(P<0.0001)$, oxidative phosphorylation $(P<0.0001)$, and tricarboxylic acid (TCA) cycle II $(P<0.0001)$, amino acid metabolism via valine and isoleucine metabolism $(P<0.0001)$. The directionality of the DEG relating to mitochondrial dysfunction, oxidative phosphorylation, TCA, valine and isoleucine degradation suggest upregulation of these pathways in the high treatment in comparison to the low treatment animals.

A total of 25 networks, regarded as having biological significance were identified. Particular networks of interest including energy production, lipid metabolism and small molecule biochemistry (Network 4; Table 4) were identified as being enriched in the high compared to the low treatment. This network highlighted 28 associated molecules, 24 of which were upregulated, and 4 of which were down

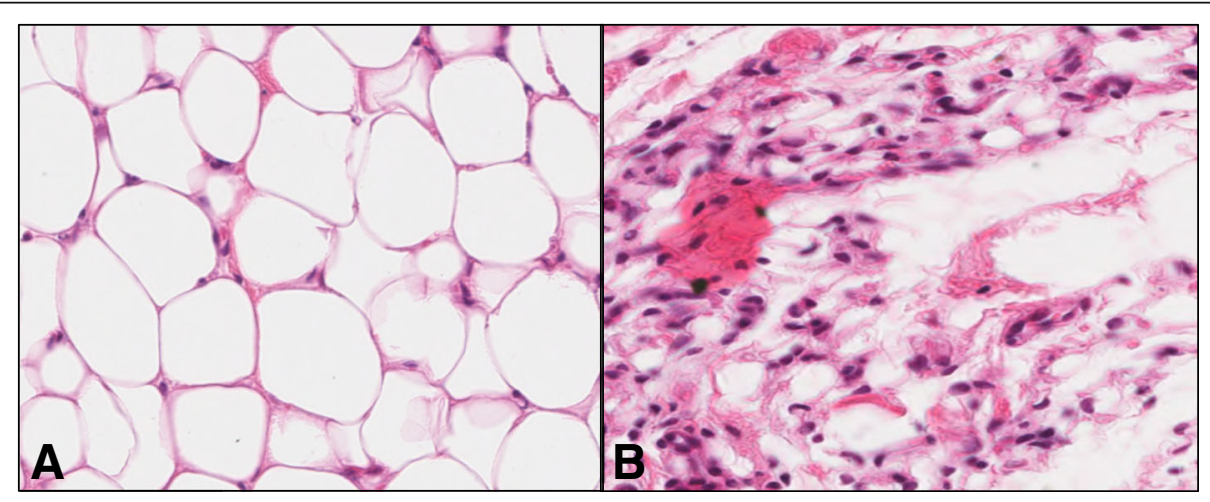

Fig. 1 Light micrograph of subcutaneous adipose tissue stained with haematoxylin and eosin. A; High plane of nutrition had large areas of adipocytes (400x), B; Low plane of nutrition had small pockets of adipocytes (400x) 


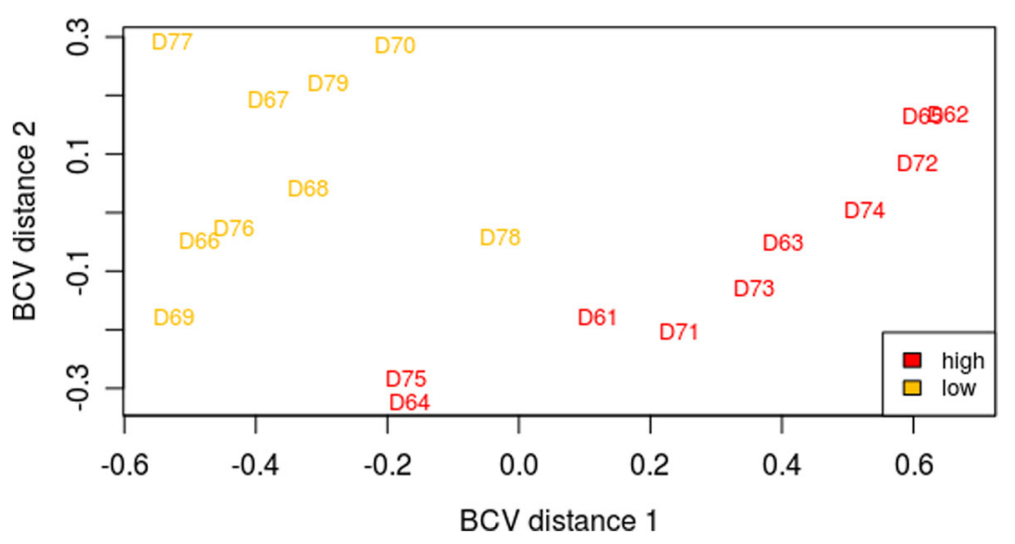

Fig. 2 Multidimensional scaling plot which shows the measured similarity of the samples in 2-dimensions. The samples labelled in yellow are the subcutaneous adipose tissue of Holstein-Friesian dairy bulls fed on a low plane of nutrition and slaughtered at 18 weeks of age and those labelled in red are the subcutaneous adipose tissue of Holstein-Friesian dairy bulls fed on a high plane of nutrition and slaughtered at 18 weeks of age. $\mathrm{BCV}=$ Biological Coefficient of Variation

Table 3 Genes differentially expressed in the subcutaneous adipose tissue of Holstein-Friesian dairy bulls fed on a low plane of nutrition in comparison to the high plane of nutrition and slaughtered at 18 weeks of age returned by Goseq $(P<0.05$; False Discovery Rate $<0.05$ : fold change $>2.0$ )

\begin{tabular}{|c|c|c|}
\hline Rank & KO Pathway & Pathway Name \\
\hline 1 & 01100 & Metabolic pathways \\
\hline 2 & 00280 & Valine, leucine and isoleucine degradation \\
\hline 3 & 00020 & Citrate Cycle -carbohydrate metabolism \\
\hline 4 & 05012 & Parkinson's disease \\
\hline 5 & 00190 & NADH:ubiquinone oxidoreductase \\
\hline 6 & 00640 & Malonate semialdehyde pathway \\
\hline 7 & 00650 & Butanoate metabolism \\
\hline 8 & 03010 & Ribosome \\
\hline 9 & 00630 & Glyoxylate and dicarboxylate metabolism \\
\hline 10 & 05010 & Alzheimer's disease \\
\hline 11 & 00071 & Fatty acid degradation \\
\hline 12 & 00620 & Pyruvate metabolism \\
\hline 13 & 00100 & Steroid biosynthesis \\
\hline 14 & 03320 & PPAR signaling pathway \\
\hline 15 & 00380 & Tryptophan metabolism \\
\hline 16 & 05016 & Huntington disease \\
\hline 17 & 00410 & beta-Alanine metabolism \\
\hline 18 & 00561 & Glycerolipid metabolism \\
\hline 19 & 04512 & ECM-receptor interaction \\
\hline 20 & 04146 & Peroxisome \\
\hline 21 & 00120 & Primary bile acid biosynthesis \\
\hline 22 & 00330 & Arginine and proline metabolism \\
\hline 23 & 00010 & Glycolysis / Gluconeogenesis \\
\hline
\end{tabular}

Rank = significance relative position $(1$ is the most significant, 23 is the least significant) KO KEGG Orthology regulated in the high compared to the low treatment animals. Of particular interest, $A D I P O Q$ (adiponectin; - 1.62 $\log$ fold change) and its receptor ADIPOR2 (-1.772 log fold change) and $L E P$ (leptin; - 4.541 log fold change) were upregulated in the high compared to the low treatment. Using the Molecule Activity Predictor (MAP) function in IPA insulin has been predicted to be inhibited in the low compared to the high treatment (Additional file 3).

\section{Lipogenesis and adipogenesis}

Animals on the low plane of nutrition had a lower of expression of MLXIPL (MLX interacting protein-like, also known as ChREBP; - 1.375 log fold change), a transcription factor that regulates lipogenesis and adipogenesis, in comparison to the high plane of nutrition. Target genes linked with adipocyte differentiation were down regulated in the low treatment in comparison to the high treatment, such as DGAT2 (diacylglycerol acyltransferase 2; - 5.215 log fold change), FASN (fatty acid synthetase; 5-184 log fold change), ACACA (acetyl-CoA carboxylase$\alpha ;-3.432 \log$ fold change), LPL (lipoprotein lipase; $3.241 \log$ fold change), PLIN1 (Perilipin $1 ;-2.279 \log$ fold change) and FABP4 (fatty acid-binding protein $4 ;-2.273$ $\log$ fold change), INSIG-1 (insulin-induced gene $1 ;-3.216$ log fold change) and GDF-10 (growth differentiation factor $10 ;-2.014)$. Genes that act as inhibitors for adipogenesis such as GATA2 (GATA binding protein 2; $1.151 \log$ fold change) and WNT2B (Wnt family member $2 \mathrm{~B} ; 1.672 \log$ fold change) were up regulated in the low plane of nutrition compared to the high.

\section{Discussion}

This is the first study to apply next generation sequencing technology to examine the effect of early calf-hood nutrition on the transcriptomic profile of subcutaneous 
Table 4 Network of biological interest generated through network analysis using Ingenuity Pathway Analysis (IP A) of subcutaneous adipose tissue of Holstein-Friesian dairy bulls fed on low plane of nutrition in comparison to a high plane of nutrition, slaughtered at 18 weeks of age

\begin{tabular}{lllll}
\hline Network ID & Top Functions & Effected Molecules in Network & Score & Focus Molecule \\
\hline 4 & Energy Production, Lipid Metabolism, & ABLIM2, ACAT1, ADIPOQ, ADIPOR2, ALDOA, BCAT2, CISD1, & 39 & 28 \\
& Small Molecule Biochemistry & CKB, FABP4, FITM2, GPD1, HOMER2, LEP, LGALS12, MT-ND1, & \\
& MT-ND6, NDUFB5, NDUFB8, NDUFS2, NDUFV1, NNAT, NR4A1, & \\
& SNCA, SORBS1, SYNE2, TUSC5 & \\
\hline
\end{tabular}

Molecules highlighted in bold are downregulated in the low plane of nutrition in comparison to the high plane of nutrition

adipose in calves. The histological and mRNA expression data indicate much lower adiposity (number and size of adipocytes) and greater number of preadipocytes in the calves subjected to the low plane of nutrition. This was consistent with evidence for the high plane of nutrition inducing an increase in both energy production and amino acid metabolic pathways in comparison to the low treatment. The 'energy production, lipid metabolism and small molecule biochemistry network' was also found to be enriched in the high compared to the low group with certain genes of particular interest within this network, including $A D I P O Q, A D I P O R 2$ and $L E P$ all consistent with increased adipogenesis and all upregulated in the high treatment group.

\section{Lipogenesis and adipogenesis}

It has been postulated that rapid growth during the post weaning period in humans can cause preadipocytes to enter terminal differentiation [19]. Some transcription factors have been highlighted as regulators of lipogenesis and adipogenesis at a transcriptional level, including sterol regulatory element binding factor 1 (SREBF1) and MLXIPL [20]. The calves on the low plane of nutrition in our study had lower expression of MLXIPL in comparison to their contemporaries on the high plane of nutrition, which was supported by the complementary histology data. It has been reported that MLXIPL expression levels increase during preadipocyte differentiation in humans, mice and rats; however, there was a dramatic decrease in $M L X I P L$ expression when cell differentiation took place in the absence of insulin [21, 22]. It is well accepted that insulin is a metabolically active hormone responsible for anabolic processes promoting growth, development, and nutrient homeostasis [23, 24]. Indeed we recorded greater plasma concentrations of insulin in the high compared with the low treatment groups at the time of slaughter (data not presented), although no differences were observed at the mRNA level. Insulin-induced gene-1 (INSIG-1) has been reported to mediate sterol regulatory element binding protein-1c (SREBP-c) which is a transcription factor involved in the regulation of the effect of insulin on lipid metabolism and adipogensis [25]. Studies have indicated that INSIG-1 increases in the fat tissue of mice developing diet induced obesity and also in differentiating 3 T3-L1 preadipocytes [26]. This highlights the importance of insulin in lipogenesis.

In a recent study [27] the authors reported a transcriptomic analysis of adipogenesis in human adipocyte tissue and identified a variety of genes as general adipocyte markers such as FABP4, PLIN1, LPL and ADIPOQ. These genes had increased expression during adipogenic differentiation consistent with the findings of others [28]. Upregulation of FABP4, FASN, ACACA and DGAT2 mRNA, which are target genes of $P P A R \gamma$, are required for fatty acid biosynthesis and adipocyte differentiation $[19,29]$. FASN is critical in the process of synthesising long chain saturated fatty acids and its function is inhibited by fasting [30]. Down regulation of $A C A C A$ has been reported in calorie restriction in adipose and liver tissue in pigs [31] and muscle in cattle [32]. It has been hypothesised that leptin controls adipocyte size via influencing DGAT expression [33]. Eventhough, no difference in PPARy expression was determined in our study, downstream genes affected by $P P A R y$ were downregulated in tissue from the animals on the low plane of nutrition. There was no evidence of $B M P$ (bone morphogenic protein), expression being affected by plane of nutrition. However, GDF10 which is linked to $B M P 3$ was found to downregulated on the low plane of nutrition. As well as a down regulation in genes that stimulate adipogenesis, in our study we found an upregulation in a key gene found to inhibit adipogenesis in the low plane of nutrition compared to the high plane of nutrition. It has been reported that GATA2 is expressed in preadipocytes and is down-regulated during terminal differentiation [34]. Studies in mouse preadipocytes show that GATA2 inhibits adipogenesis and traps cells at preadipocyte stage by direct suppression of PPAR $\gamma$ [34]. In addition, the histological data showing much lower adiposity (number and size of adipocytes) and greater number of preadipocytes in the animals on the low plane of nutrition corroborates the transcriptomic data aforementioned.

\section{Endocrine regulatory networks}

Insulin plays a role in regulating the synthesis and secretion of leptin, as adipocytes cultured in the presence of insulin increased the synthesis and secretion of leptin $[35,36]$. It is therefore unsurprising that IPA predicted, when applied to our data, the stimulation of insulin as 
well as the known upregulation of LGALS12 (Galectin 12), BCAT2 (Branched Chain Amino Acid Transaminase 2) and $A D I P O Q$ (adiponection), which are all located upstream of $L E P$ and likely resulted in $L E P$ to be upregulated in the high treatment. Plasma leptin is positively correlated with body fat in growing cattle [37] where it has been shown to signal energy abundance by informing the various bodily systems, including the HPT axis, of the prevailing metabolic status and energy reserves via circulating leptin protein concentrations [12]. For example, in adult animals, studies which examined the adipose tissue transcriptome of pre-partum dairy cows have shown that cows with greater BCS had greater expression of $L E P$ [38]. Consistent with this we found that calves on the high plane of nutrition in our study had $>4.5$ log fold higher LEP expression compared with animals the low animals. Despite this, when plasma concentrations were assayed, we failed to find any difference in leptin protein concentrations between the two groups (English et al., unpublished). This is in agreement with studies in both young dairy and beef bulls $[1,2,39]$. The lack of differences in plasma leptin concentrations may be due to the minute quantity of adipose tissue laid down in calves at this stage of development and/or the relative insensitivity of protein immunoassays compared with transcript based molecular approaches.

Leptin does not directly affect GnRH neurons of the hypothalamus and knocking out of leptin receptors on the GnRH neurons does not apparently delay the onset of puberty nor indeed affect subsequent fertility [13]. However, leptin has been found to indirectly influence the timing of puberty via the regulation of the hypothalamic Kiss1 neurons' stimulation of GnRH [10]. Leptin can also bypass the hypothalamus and act on the pituitary gland and the testes; with leptin modestly stimulating gonadotropin secretion at a pituitary level and both direct stimulatory and inhibitory actions of leptin been reported in the gonads [40]. At high leptin concentrations, it has been reported to inhibit testosterone secretion in vitro in adult rats but to have no effect in pubertal rats [41]. This is possibly due to the fact that pubertal testes produce more $5 \alpha$-reduced androgens than testosterone [42]. In research carried out by Byrne et al. (2016) bulls which had experienced dietary restriction prior to 6 months of age and an increase in plane of nutrition post 6 months of age leading to an increase in leptin concentration, were older at puberty than their contemporaries offered a high plane of nutrition; highlighting the importance of early life nutrition on age at puberty.

Adiponectin functions in the regulation of lipid and glucose metabolism, insulin sensitivity as well as inflammation [43-45]. Its concentration in blood has been shown to be negatively correlated to adipocyte size [46]. Food intake inhibits ADIPOQ mRNA expression in WAT and therefore, inhibits adiponectin concentration in serum [47]. Globular adiponectin protein has been found to inhibit the secretion of GnRH in GT1-7 cells derived from mouse hypothalamic GnRH neurons via the mediation of adenosine monophosphate-activated protein kinase (AMPK; Wen et al., 2008). However, high doses of adiponectin in MA-10 mouse Leydig cells has been reported to advance progesterone production through an increase in StAR (Steroidogenic Acute Regulatory Protein) and the CYP11A1 (Cytochrome P450 Family 11 Subfamily A Member 1) steroidogenesis enzyme, suggesting that adiponectin could stimulate testosterone production from the Leydig cells [48]. It has also been shown that adiponectin reduces LH secretion directly from the gonadotropes in the anterior pituitary via AMPK [49]. Pre-pubertal children with increased body mass index (BMI), though not classified as obese, had lower adiponectin concentration in subcutaneous adipose [50]. Similarly, postpartum cows, in negative energy balance had decreased ADIPOR1 and ADIPOR2 abundance in subcutaneous adipose compared to antepartum cows [51] which is in agreement with our findings with calves where we found $A D I P O Q$ and its receptor $A D I P O R 2$ were downregulated in the low compared to the high treatment groups. It has been reported that ADIPOR2 null mice demonstrated seminiferous tubules with aspermia but had normal testosterone concentration [52]. It has also been found that the abundance of $A D I P O Q$, ADIPOR1 and ADIPOR2 is greater in high fertility compared to that of medium and low fertility Holstein bulls [53]. There was no difference in serum concentrations of adiponectin between the two groups in the current study (English et al., unpublished), which like leptin may be due to the overall low adipose deposition in young bull calves. Using a very similar animal model and the same assays as those employed here Byrne et al. (2016); similarly observed in leptin and adiponectin concentrations during the same development as reported here. However, from 8 months on age onwards, bull calves offered a high plane of nutrition had greater leptin compared to their contemporaries on a moderate plane of nutrition (ADG c. $0.65 \mathrm{~kg} /$ day).

\section{Branched chain amino acid metabolism}

Branched chain amino acids (BCAAs), leucine, isoleucine and valine are essential amino acids and, unlike other amino acids which are catabolized by the liver, BCAAs are catabolized mainly in the muscle, adipose, kidney and the brain; as the liver does not contain the branched amino acid transferase enzyme (BCAT2), necessary for BCAA catabolism [54]. The isoleucine/valine degradation pathway enters the TCA cycle either directly or via an acetyl derived intermediate and in this study we found that genes involved in the valine degradation pathway encoding $B C A T 2$, 2-oxoisovalerate dehydrogenase (BCKDHB and 
Table 5 Oxidative Phosphorylation genes differentially expressed in the subcutaneous adipose tissue of the Holstein-Friesian dairy bulls feed on a low plane of nutrition in comparison to the high plane of nutrition and slaughtered at 18 weeks of age

\begin{tabular}{|c|c|c|}
\hline Symbol & Gene Name & Log Fold Change \\
\hline \multicolumn{3}{|l|}{ Complex I } \\
\hline NDUFA4 & NDUFA4, mitochondrial complex associated & -1.472 \\
\hline NDUFA5 & NADH:ubiquinone oxidoreductase subunit A5 & -1.117 \\
\hline NDUFA8 & NADH:ubiquinone oxidoreductase subunit A8 & -1.134 \\
\hline NDUFA9 & NADH:ubiquinone oxidoreductase subunit A9 & -1.202 \\
\hline NDUFA12 & NADH:ubiquinone oxidoreductase subunit A12 & -1.221 \\
\hline NDUFB3 & NADH:ubiquinone oxidoreductase subunit B3 & -1.401 \\
\hline NDUFB5 & NADH:ubiquinone oxidoreductase subunit B5 & -1.269 \\
\hline NDUFB8 & NADH:ubiquinone oxidoreductase subunit B8 & -1.137 \\
\hline NDUFS1 & NADH:ubiquinone oxidoreductase core subunit S1 & -1.711 \\
\hline NDUFS2 & NADH:ubiquinone oxidoreductase core subunit S2 & -1.464 \\
\hline NDUFS4 & NADH:ubiquinone oxidoreductase subunit S4 & -1.196 \\
\hline NDUFS7 & NADH:ubiquinone oxidoreductase core subunit S7 & -1.494 \\
\hline NDUFS8 & NADH:ubiquinone oxidoreductase core subunit $\mathbf{8} 8$ & -1.328 \\
\hline NDUFV1 & NADH:ubiquinone oxidoreductase core subunit V1 & -1.703 \\
\hline MT-ND1 & NADH dehydrogenase, subunit 1 (complex I) & -1.058 \\
\hline MT-ND2 & MTND2 & -1.182 \\
\hline MT-ND3 & NADH dehydrogenase, subunit 3 (complex I) & -1.035 \\
\hline MT-ND4 & NADH dehydrogenase, subunit 4 (complex I) & -1.522 \\
\hline MT-ND5 & NADH dehydrogenase, subunit 5 (complex I) & -1.848 \\
\hline MT-ND4L & NADH dehydrogenase, subunit $4 \mathrm{~L}$ (complex I) & -1.552 \\
\hline \multicolumn{3}{|l|}{ Complex III } \\
\hline UQCR10 & ubiquinol-cytochrome $\mathrm{c}$ reductase, complex III subunit X & -1.629 \\
\hline UQCR11 & ubiquinol-cytochrome $\mathrm{c}$ reductase, complex III subunit XI & -1.217 \\
\hline UQCRB & ubiquinol-cytochrome $\mathrm{c}$ reductase binding protein & -1.498 \\
\hline UQCRC1 & ubiquinol-cytochrome c reductase core protein I & -1.479 \\
\hline UQCRC2 & ubiquinol-cytochrome c reductase core protein ॥ & -1.532 \\
\hline MT-CYB & cytochrome b & -1.647 \\
\hline \multicolumn{3}{|l|}{ Complex IV } \\
\hline $\operatorname{cox} 5 \mathrm{~A}$ & cytochrome c oxidase subunit $5 \mathrm{~A}$ & -1.567 \\
\hline COX5B & cytochrome c oxidase subunit 5B & -1.566 \\
\hline COX6B1 & cytochrome c oxidase subunit 6B1 & -1.049 \\
\hline cox6C & cytochrome c oxidase subunit Vlc & -1.115 \\
\hline COX7A2 & cytochrome c oxidase subunit 7A2 & -1.061 \\
\hline CYB5A & cytochrome b5 type $\mathrm{A}$ & -1.559 \\
\hline $\mathrm{CYC1}$ & cytochrome c1 & -1.072 \\
\hline CYCS & cytochrome c, somatic & -1.169 \\
\hline MT-CO1 & cytochrome c oxidase subunit I & -1.695 \\
\hline MT-CO2 & cytochrome c oxidase subunit II & -1.39 \\
\hline MT-CO3 & cytochrome c oxidase III & -1.161 \\
\hline \multicolumn{3}{|l|}{ Complex V } \\
\hline ATP5A1 & ATP synthase, $\mathrm{H}+$ transporting, mitochondrial F1 complex, alpha subunit 1, cardiac muscle & -1.339 \\
\hline ATP5B & ATP synthase, $\mathrm{H}+$ transporting, mitochondrial F1 complex, beta polypeptide & -1.205 \\
\hline
\end{tabular}


Table 5 Oxidative Phosphorylation genes differentially expressed in the subcutaneous adipose tissue of the Holstein-Friesian dairy bulls feed on a low plane of nutrition in comparison to the high plane of nutrition and slaughtered at 18 weeks of age (Continued)

\begin{tabular}{cll}
\hline Symbol & Gene Name & Log Fold Change \\
\hline ATP5C1 & ATP synthase, H+ transporting, mitochondrial F1 complex, gamma polypeptide 1 & -1.186 \\
ATP5D & ATP synthase, H+ transporting, mitochondrial F1 complex, delta subunit & -1.246 \\
ATP5G1 & ATP synthase, H+ transporting, mitochondrial Fo complex subunit C1 (subunit 9) & -1.183 \\
ATP5G3 & ATP synthase, H+ transporting, mitochondrial Fo complex subunit C3 (subunit 9) & -1.548 \\
ATP5J2 & ATP synthase, H+ transporting, mitochondrial Fo complex subunit F2 & -1.205 \\
ATP5O & ATP synthase, H+ transporting, mitochondrial F1 complex, O subunit & -1.073 \\
MT-ATP6 & ATP synthase F0 subunit 6 & -1.22 \\
\hline
\end{tabular}

$D L D)$, 2-methylacyl-CoA dehydrogenase (ACADSB), enoyl-CoA hydratase (ECHS1, EHHADH, HADHA and $H A D H B)$, 3-hydroxyisobutyryl-CoA hydrolase $(H I B C H)$, methylmalonate-semialdehyde dehydrogenase (ALDH6A1) and (S)-3-amino-2-methylpropionate transaminase (ABAT) were all down regulated in the low in contrast to the high treatment. Similarly, within the isoleucine degradation pathway, genes encoding for branched-chain-aminoacid transaminase (BCAT2), branched-chain $\alpha$-keto acid dehydrogenase complex $(D L D)$, 2-methylacyl-CoA dehydrogenase $(A C A D S B)$, enoyl-CoA hydratase (ECHS1, EHHADH, $H A D H A$ and $H A D H B)$, 3-hydroxy-2-methybutyryl-CoA dehydrogenase (HSD17B10) and acetyl-CoA $\mathrm{C}$-acetyltransferase $(A C A T 1)$ were down regulated in the low compared to the high treatment. Expression of genes involved in branched chain amino acids metabolism were upregulated during 3 T3-L1 adipocyte differentiation $[55,56]$ suggesting that BCAA are involved in fatty acid synthesis [57]. It has also been shown that BCAA, especially leucine, systemic concentrations increase leptin secretion in vitro in murine [58] and decreases food intake in vitro in rats [59]. Systemic concentrations of branched chain amino acid are elevated in response to over nutrition in children and adolescents [60]. Therefore, it is reasonable to suggest that the evidence for a decline in BCAA degradation may be a result of the moderate restriction in dietary allowance experienced by our low plane of nutrition calves. The BCAA catabolic by-products mentioned in this study are intended for integration into the TCA cycle and therefore, any down regulation of the BCAA degradation pathway would cause a decrease in mitochondrial respiration in existing adipocytes [61].

\section{Energy production and mitochondrial dysfunction}

Mitochondria are key organelles in cellular energetics as they catabolise carbohydrates, lipids and proteins to synthesise ATP and metabolites for not only growth but also adipocyte differentiation and maturation [62]. There are four stages involved in aerobic respiration. Glycolysis occurs in the cytoplasm and the remaining steps, pyruvate oxidation, the TCA cycle and electron transport chain and chemiosmosis (as employed in oxidative phosphorylation) take place within the inner membrane of the mitochondria [63]. The two pyruvate molecules, the products of glycolysis, enter the mitochondrion and are converted to acetyl coenzyme A (acetyl CoA). Acetyl CoA enters the TCA cycle which drives the cycle to produce NADH, ATP and $\mathrm{FADH}_{2}$. Genes encoding for citrate synthase $(C S)$ namely, aconitase (ACO1 and $A C O 2)$, isocitrate dehydrogenase $(I D H 3 B), \alpha$-ketoglutarate dehydrogenase (OGDH and $D L D)$, succinyl-CoA synthetase (SUCLG1), fumarase $(F H)$ and malate dehydrogenase $(M D H 1)$, were all found to be down regulated in the low compared to the high treatment. NADH and $\mathrm{FADH}_{2}$ donate electrons to the electron transport chain, which powers ATP synthesis via oxidative phosphorylation. Genes associated with NADH dehydrogenase Complex I, succinate dehydrogenase Complex II, ubiquinol-cyt c oxidoreductase Complex III, cytochrome c oxidase Complex IV and ATP synthase Complex V were down regulated in the low plane of nutrition compared to the high plane of nutrition (Table 5). It has been reported that there is an increase in mRNA expression of nucleus-encoded mitochondrial genes for enzymes such as pyruvate carboxylase and pyruvate dehydrogenase complex during differentiating 3 T3-L1 adipocytes [64]. Pyruvate carboxylase has been found to be overexpressed upon conversion of preadipocytes to adipocytes [65]. This is consistent with the results of our study where the high plane of nutrition calves were at a more advanced stage of adipocyte differentiation, at tissue recovery, as indicated by the significant number of adipocytes following histological investigation when compared with their lower nutrition counterparts.

\section{Conclusion}

This study clearly demonstrates that the prevailing plane of nutrition of bull calves during the first 18 weeks of life can have a significant effect on the transcriptional and morphological functionality of subcutaneous adipose tissue. A high plane of nutrition during early calf hood has been shown to positively affect adipokines, leptin 
and adiponectin whose functions include signalling metabolic status to the reproductive system. The data generated in this study broaden our knowledge base around the potential molecular processes underlying the biological cross-talk between peripheral tissues that may be metabolic mediators of important events such as timing of puberty onset etc. Furthermore, global differential gene expression patterns provide data which may have implications for the selection of robust biomarkers to identify animals with superior genetic potential for early pubertal onset, greater carcass adiposity and other economically important traits, shown to be latently affected by early life plane of nutrition.

\section{Additional files}

Additional file 1: Molecular and cellular function of differentially expressed genes of the subcutaneous adipose tissue of Holstein-Friesian dairy bulls fed on a low versus high plane of nutrition and slaughtered at 18 weeks of age. The bars indicate the likelihood $[-\log (P$-value)] that the specific molecular and cellular function was affected by a high plane of nutrition. The threshold line in the bar chart represents a $p$-value of 0.05 . (PDF $1520 \mathrm{~kb}$ )

Additional file 2: Biochemical pathway significantly enriched in the subcutaneous adipose tissue of Holstein-Friesian dairy bulls fed on a low plane of nutrition in comparison to a high plane of nutrition, slaughtered at 18 weeks of age. Greens bars represent genes down regulated and red bars up regulated genes as percentages of the overall number of genes in each pathway. The significance of each pathway is represented by the orange line describing -log(p-value). The numbers along the right hand side indicate the number of genes in total involved in each pathway. The $P$-value is calculated by the number of genes from our dataset of differentially expressed genes that participate in a particular pathway and dividing it by the total number of genes in the Canonical Pathway in Ingenuity Pathways Analysis (IPA) analysis. (PDF $4096 \mathrm{~kb}$ )

Additional file 3: Genes differentially expressed in the subcutaneous adipose of Holstein-Friesian dairy bulls fed on low plane of nutrition in comparison to high plane of nutrition, slaughtered at 18 weeks of age. Genes outlined by the Molecule Activity Predictor function in IPA. (XLS $106 \mathrm{~kb}$ )

\section{Abbreviations \\ ACACA: Acetyl-CoA carboxylase-a; ADIPOQ: Adiponectin; AMPK: Adenosine monophosphate-activated protein kinase; BCAA: Branched chain amino acids; BCAT2: Branched chain amino acid transaminase 2; BCS: Body conditioning score; BMI: Body mass index; BMP: Bone morphogenic bone; bp: Base pairs; CYP11A1: Cytochrome P450 Family 11 Subfamily A Member 1; \\ DEG: Differentially expressed genes; DGAT2: Diacylglycerol acyltransferase 2; FABP4: Fatty acid-binding protein 4; FASN: Fatty acid synthetase; FDR: False discovery rate; GATA2: GATA binding protein 2; GDF-10: Growth differentiation factor 10; GnRH: Gonadotropin releasing hormone; H\&E: Haematoxylin and eosin; HPT: Hypothalamic-pituitary-testicular; INSIG-1: Insulin-induced gene 1; IPA: Ingenuity pathway analysis; KEGG: Kyoto Encyclopaedia of genes and genomes; KISS1: Kisspeptin; LEP: Leptin; LGALS12: Galectin 12; LH: Luteinising hormone; LPL: Lipoprotein lipase; MAP: Molecule activity predictor; MDS: Multi-dimensional scaling; MLXIPL/ChREBP: MLX interacting protein-like; PLIN1: Perilipin 1; RIN: RNA integrity number; RNAseq: RNA sequencing; SREBF1: Sterol regulatory element binding factor 1; SREBP-c: Sterol regulatory element binding protein-1c; STAR: Spliced transcripts alignment to a reference; StAR: Steroidogenic acute regulatory protein; TCA: Tricarboxylic acid; WAT: White adipose tissue; WNT2B: Wnt family member 2B}

\section{Acknowledgments}

The contribution of Dr. M. McCabe in the preperation of the RNAseq libraries as well as that of the farm staff at Teagasc, Grange Beef Research Centre, for care and management of the animals is gratefully acknowledged.

\section{Funding}

This project was jointly funded by the Irish Department of Agriculture, Food and the Marine under the Research Stimulus Fund (Ref: 11/S/116) and the Irish Research Council (GOIPG/2013/1391). Neither funding body was involved in the design of the study and collection, analysis, and interpretation of data and in writing of this manuscript.

\section{Availability of data and materials}

The dataset generated during and analysed in the current study are available in the NCB's Gene Expression Omnibus repository, and are accessible through GEO Series accession number GSE97674 [https:/www.ncbi.nlm.nih.gov/gds/?term=GSE97674].

\section{Authors' contributions}

Author contributions: AME, SMW, SF and DAK conception and design of research; $A M E$ and $C B$ performed experiments; $A M E$ and $P C$ analysed data; $A M E$ and DAK interpreted results of experiments; AME prepared figures; AME drafted manuscript; AME, CB, PC, SMW, SF and DAK edited and revised manuscript; $A M E, C B, P C, S M W, S F$ and DAK approved final version of manuscript.

\section{Ethics approval}

All procedures involving animals were approved by the Teagasc Animal Ethics Committee (TAEC30/2013) and were licensed by the Health Products Regulatory Authority, Ireland in accordance with the European Union Directive 2010/36/EU.

\section{Competing interests}

The authors declare that they have no competing interests.

\section{Publisher's Note}

Springer Nature remains neutral with regard to jurisdictional claims in published maps and institutional affiliations.

\section{Author details}

${ }^{1}$ Animal and Bioscience Research Department, Teagasc Grange, Dunsany, Co. Meath C15 PW93, Ireland. '2aboratory of Animal Reproduction, Department of Biological Sciences, School of Natural Sciences, Faculty of Science and Engineering, University of Limerick, Limerick V94 T9PX, Ireland. ${ }^{3}$ School of Agriculture and Food Science, University College Dublin, Belfield, Dublin 4, Dublin D04 N2E5, Ireland.

Received: 11 August 2017 Accepted: 16 April 2018

Published online: 24 April 2018

\section{References}

1. Brito LF, Barth AD, Rawlings NC, Wilde RE, Crews DH Jr, Boisclair YR, Ehrhardt RA, Kastelic JP. Effect of feed restriction during calfhood on serum concentrations of metabolic hormones, gonadotropins, testosterone, and on sexual development in bulls. Reproduction. 2007;134(1):171-81.

2. Dance A, Thundathil J, Wilde R, Blondin P, Kastelic J. Enhanced early-life nutrition promotes hormone production and reproductive development in Holstein bulls. J Dairy Sci. 2015;98(2):987-98.

3. Byrne CJ, Fair S, English AM, Urh C, Sauerwein H, Crowe MA, Lonergan P, Kenny DA. Plane of nutrition before and after 6 months of age in HolsteinFriesian bulls: II. Effects on metabolic and reproductive endocrinology and identification of physiological markers of puberty and sexual maturation. J Dairy Sci. 2018;101(4):3460-75.

4. Rawlings N, Evans AC, Chandolia RK, Bagu ET. Sexual maturation in the bull. Reprod Domest Anim. 2008;43(Suppl 2):295-301.

5. Jin W, Olson EN, Moore SS, Basarab JA, Basu U, Guan LL. Transcriptome analysis of subcutaneous adipose tissues in beef cattle using $3^{\prime}$ digital gene expression-tag profiling. J Anim Sci. 2012;90(1):171-83.

6. Romao JM, He ML, McAllister TA, Guan LL. Effect of age on bovine subcutaneous fat proteome: molecular mechanisms of physiological variations during beef cattle growth. J Anim Sci. 2014;92(8):3316-27.

7. Graugnard DE, Berger LL, Faulkner DB, Loor JJ. High-starch diets induce precocious adipogenic gene network up-regulation in longissimus lumborum of early-weaned Angus cattle. Brit J Nutr. 2010;103(7):953-63.

8. Wang YH, Bower NI, Reverter A, Tan SH, De Jager N, Wang R, McWilliam SM, Cafe LM, Greenwood PL, Lehnert SA. Gene expression patterns during intramuscular fat development in cattle. J Anim Sci. 2009;87(1):119-30. 
9. Landry D, Cloutier F, Martin LJ. Implications of leptin in neuroendocrine regulation of male reproduction. Reprod Bio. 2013;13(1):1-14.

10. Sanchez-Garrido MA, Tena-Sempere M. Metabolic control of puberty: roles of leptin and kisspeptins. Hormones Behav. 2013;64(2):187-94.

11. Ouchi N, Parker JL, Lugus JJ, Walsh K. Adipokines in inflammation and metabolic disease. Nat Rev Immunol. 2011:11(2):85-97.

12. Ahima RS, Saper CB, Flier JS, Elmquist JK. Leptin regulation of neuroendocrine systems. Front Neuroendocrinol. 2000;21(3):263-307.

13. Quennell JH, Mulligan AC, Tups A, Liu X, Phipps SJ, Kemp CJ, Herbison AE, Grattan DR, Anderson GM. Leptin indirectly regulates gonadotropinreleasing hormone neuronal function. Endocrinology. 2009;150(6):2805-12.

14. Smith JT, Acohido BV, Clifton DK, Steiner RA. KiSS-1 neurones are direct targets for leptin in the Ob/Ob mouse. J Neuroendocrinol. 2006;18(4):298-303.

15. Tabandeh MR, Hosseini A, Sarkoohi P, Seifi S. Changes in the gene expression of adiponectin and adiponectin receptors (AdipoR1 and AdipoR2) in pituitary and hypothalamus during the estrous cycle and pregnancy. Clin Biochem. 2011;44(13):S303.

16. Aksglaede L, Juul A, Olsen LW, Sørensen TI. Age at puberty and the emerging obesity epidemic. PLoS One. 2009;4(12):e8450.

17. Young MD, Wakefield MJ, Smyth GK, Oshlack A. Gene ontology analysis for RNA-seq: accounting for selection bias. Genome Biol. 2010;11(2):R14.

18. Kanehisa M, Goto S. KEGG: Kyoto encyclopedia of genes and genomes. Nucleic Acids Res. 2000;28(1):27-30.

19. Hausman GJ, Dodson MV, Ajuwon K, Azain M, Barnes KM, Guan LL, Jiang Z, Poulos SP, Sainz RD, Smith S, et al. Board-invited review: the biology and regulation of preadipocytes and adipocytes in meat animals. J Anim Sci. 2009;87(4):1218-46.

20. Loor JJ. Transcriptomics of muscle and fat development in growing cattle. In: Crovetto GM, editor. Energy and protein metabolism and nutrition. Wageningen: Wageningen Academic Publishers; 2010. p. 59-68.

21. Hurtado del Pozo C, Vesperinas-García G, Rubio M-Á, Corripio-Sánchez R, Torres-García AJ, Obregon M-J, Calvo RM. ChREBP expression in the liver, adipose tissue and differentiated preadipocytes in human obesity. BBA-Mol Cell Bio L. 2011;1811(12):1194-200.

22. He Z, Jiang T, Wang Z, Levi M, Li J. Modulation of carbohydrate response element-binding protein gene expression in 3T3-L1 adipocytes and rat adipose tissue. Am J Physiol Endoc M. 2004;287(3):424-30.

23. Taniguchi CM, Emanuelli B, Kahn CR. Critical nodes in signalling pathways: insights into insulin action. Nat Rev Mol Cell Bio. 2006;7(2):85-96.

24. Cheng $Z$, Tseng $Y$, White MF. Insulin signaling meets mitochondria in metabolism. Trends Endocrin Meta. 2010;21(10):589-98.

25. Dong XY, Tang SQ. Insulin-induced gene: a new regulator in lipid metabolism. Peptides. 2010;31(11):2145-50.

26. Li J, Takaishi K, Cook W, McCorkle SK, Unger RH. Insig-1 "brakes" lipogenesis in adipocytes and inhibits differentiation of preadipocytes. Proc Natl Acad Sci U S A. 2003;100(16):9476-81.

27. Ambele MA, Dessels C, Durandt C, Pepper MS. Genome-wide analysis of gene expression during adipogenesis in human adipose-derived stromal cells reveals novel patterns of gene expression during adipocyte differentiation. Stem Cell Res. 2016;16(3):725-34.

28. Ullah M, Stich S, Haupl T, Eucker J, Sittinger M, Ringe J. Reverse differentiation as a gene filtering tool in genome expression profiling of adipogenesis for fat marker gene selection and their analysis. PLoS One. 2013;8(7):e69754.

29. Obregon M-J. Thyroid hormone and adipocyte differentiation. Thyroid. 2008; 18(2):185-95.

30. Wakil SJ. Fatty acid synthase, a proficient multifunctional enzyme. Biochemistry. 1989;28(11):4523-30.

31. Lkhagvadorj S, Qu L, Cai W, Couture OP, Barb CR, Hausman GJ, Nettleton D, Anderson LL, Dekkers JC, Tuggle CK. Gene expression profiling of the shortterm adaptive response to acute caloric restriction in liver and adipose tissues of pigs differing in feed efficiency. Am J Physiol Reg Integrat Comp Physiol. 2010;298(2):R494-507.

32. Keogh K, Kenny DA, Cormican P, McCabe MS, Kelly AK, Waters SM. Effect of dietary restriction and subsequent re-alimentation on the transcriptional profile of bovine skeletal muscle. PLoS One. 2016;11(2):e0149373.

33. Suzuki R, Tobe K, Aoyama M, Sakamoto K, Ohsugi M, Kamei N, Nemoto S, Inoue A, Ito Y, Uchida S, et al. Expression of DGAT2 in white adipose tissue is regulated by central leptin action. J Biol Chem. 2005;280(5):3331-7.

34. Tong $\mathrm{Q}$, Dalgin G, Xu H, Ting CN, Leiden JM, Hotamisligil GS. Function of GATA transcription factors in preadipocyte-adipocyte transition. Science. 2000;290(5489):134-8.
35. Kim JB, Sarraf P, Wright M, Yao KM, Mueller E, Solanes G, Lowell BB, Spiegelman BM. Nutritional and insulin regulation of fatty acid synthetase and leptin gene expression through ADD1/SREBP1. J Clin Invest. 1998;101(1):1-9.

36. Barr VA, Malide D, Zarnowski MJ, Taylor SI, Cushman SW. Insulin stimulates both leptin secretion and production by rat white adipose tissue. Endocrinology. 1997;138(10):4463-72.

37. Ehrhardt R, Slepetis R, Siegal-Willott J, Van Amburgh M, Bell A, Boisclair Y. Development of a specific radioimmunoassay to measure physiological changes of circulating leptin in cattle and sheep. J Endocrinol. 2000;166(3): $519-28$.

38. Vailati-Riboni M, Kanwal M, Bulgari O, Meier S, Priest NV, Burke CR, Kay JK, McDougall S, Mitchell MD, Walker CG, et al. Body condition score and plane of nutrition prepartum affect adipose tissue transcriptome regulators of metabolism and inflammation in grazing dairy cows during the transition period. J Dairy Sci. 2016;99(1):758-70.

39. Byrne CJ, Fair S, English AM, Urh C, Sauerwein H, Crowe MA, Lonergan P, Kenny DA. Effect of breed, plane of nutrition and age on growth, scrotal development, metabolite concentrations and on systemic gonadotropin and testosterone concentrations following a GnRH challenge in young dairy bulls. Theriogenology. 2017;96:58-68.

40. Tena-Sempere M. Roles of ghrelin and leptin in the control of reproductive function. Neuroendocrinology. 2007:86(3):229-41.

41. Tena-Sempere M, Pinilla L, Gonzalez LC, Dieguez C, Casanueva FF, Aguilar E. Leptin inhibits testosterone secretion from adult rat testis in vitro. J Endocrinol. 1999;161(2):211-8.

42. Corpéchot C, Baulieu E-E, Robel P. Testosterone, dihydrotestosterone and androstanediols in plasma, testes and prostates of rats during development. Acta Endocrinol-COP. 1981;96(1):127-35.

43. Kadowaki T, Yamauchi T, Kubota N, Hara K, Ueki K, Tobe K. Adiponectin and adiponectin receptors in insulin resistance, diabetes, and the metabolic syndrome. J Clin Invest. 2006;116(7):1784-92.

44. Ahima RS, Lazar MA. Adipokines and the peripheral and neural control of energy balance. Mol Endocrinol. 2008;22(5):1023-31.

45. Galic S, Oakhill JS, Steinberg GR. Adipose tissue as an endocrine organ. Mol Cell Endocrinol. 2010;316(2):129-39.

46. Hammes TO, Costa Cdos S, Rohden F, Margis R, de Almeida JC, Padoin AV, Mottin CC, Guaragna RM. Parallel down-regulation of FOXO1, PPARgamma and adiponectin mRNA expression in visceral adipose tissue of class III obese individuals. Obesity Facts. 2012;5(3):452-9.

47. Steinberg GR, Kemp BE. Adiponectin: starving for attention. Cell Metab. 2007;6(1):3-4.

48. Landry $\mathrm{D}$, Paré $\mathrm{A}$, Jean $\mathrm{S}$, Martin $\sqcup$. Adiponectin influences progesterone production from MA-10 Leydig cells in a dose-dependent manner. Endocrine. 2015:48(3):957-67.

49. Lu M, Tang Q, Olefsky JM, Mellon PL, Webster NJG. Adiponectin activates adenosine monophosphate-activated protein kinase and decreases luteinizing hormone secretion in LBT2 Gonadotropes. Mol Endocrinol. 2008; 22(3):760-71.

50. Sabin MA, Holly JMP, Shield JPH, Turner SJ, Grohmann MJ, Stewart CEH, Crowne EC. Mature subcutaneous and visceral adipocyte concentrations of adiponectin are highly correlated in Prepubertal children and inversely related to body mass index standard deviation score. J Clin Endocrin Metab. 2006;91(1):332-5.

51. Lemor A, Hosseini A, Sauerwein H, Mielenz M. Transition period-related changes in the abundance of the mRNAs of adiponectin and its receptors, of visfatin, and of fatty acid binding receptors in adipose tissue of highyielding dairy cows. Domest Anim Endocrinol. 2009;37(1):37-44.

52. Bjursell M, Ahnmark A, Bohlooly-Y M, William-Olsson L, Rhedin M, Peng X-R, Ploj $K$, Gerdin A-K, Arnerup G, Elmgren A, et al. Opposing effects of adiponectin receptors 1 and 2 on energy metabolism. Diabetes. 2007;56(3):583.

53. Kasimanickam VR, Kasimanickam RK, Kastelic JP, Stevenson JS. Associations of adiponectin and fertility estimates in Holstein bulls. Theriogenology. 2013;79(5):766-777.e763.

54. Herman MA, She P, Peroni OD, Lynch CJ, Kahn BB. Adipose tissue branched chain amino acid (BCAA) metabolism modulates circulating BCAA levels. J Biol Chem. 2010;285(15):11348-56.

55. Frerman FE, Sabran JL, Taylor JL, Grossberg SE. Leucine catabolism during the differentiation of 3T3-L1 cells. Expression of a mitochondrial enzyme system. J Biol Chem. 1983;258(11):7087-93.

56. Kedishvili NY, Popov KM, Jaskiewicz JA, Harris RA. Coordinated expression of valine catabolic enzymes during adipogenesis: analysis of activity, mRNA, 
protein levels, and metabolic consequences. Arch Biochem Biophys. 1994; 315(2):317-22.

57. Crown SB, Marze N, Antoniewicz MR. Catabolism of branched chain amino acids contributes significantly to synthesis of odd-chain and even-chain fatty acids in 3T3-L1 adipocytes. PLoS One. 2016;10(12):e0145850.

58. Lynch CJ, Gern B, Lloyd C, Hutson SM, Eicher R, Vary TC. Leucine in food mediates some of the postprandial rise in plasma leptin concentrations. Am J Phys Endocrinol Metab. 2006;291(3):E621-30.

59. Cota D, Proulx K, Smith KA, Kozma SC, Thomas G, Woods SC, Seeley RJ. Hypothalamic mTOR signaling regulates food intake. Science. 2006; 312(5775):927-30.

60. McCormack SE, Shaham O, McCarthy MA, Deik AA, Wang TJ, Gerszten RE, Clish CB, Mootha VK, Grinspoon SK, Fleischman A. Circulating branched-chain amino acid concentrations are associated with obesity and future insulin resistance in children and adolescents. Pediatric Obesity. 2013:8(1):52-61.

61. Pietiläinen $\mathrm{KH}$, Naukkarinen J, Rissanen A, Saharinen J, Ellonen P, Keränen $\mathrm{H}$, Suomalainen A, Götz A, Suortti T, Yki-Järvinen $H$, et al. Global transcript profiles of fat in monozygotic twins discordant for BMI: pathways behind acquired obesity. PLoS Med. 2008:5(3):e51.

62. De Pauw A, Tejerina S, Raes M, Keijer J, Arnould T. Mitochondrial (dys)function in adipocyte (de)differentiation and systemic metabolic alterations. Am J Pathol. 2009;175(3):927-39.

63. Goldenthal MJ, Marin-Garcia J. Mitochondrial signaling pathways: a receiver/ integrator organelle. Mol Cell Biochem. 2004;262(1-2):1-16.

64. Wilson-Fritch L, Burkart A, Bell G, Mendelson K, Leszyk J, Nicoloro S, Czech M, Corvera S. Mitochondrial biogenesis and remodeling during adipogenesis and in response to the insulin sensitizer rosiglitazone. Mol Cell Biol. 2003;23(3):1085-94.

65. Owen OE, Kalhan SC, Hanson RW. The key role of Anaplerosis and cataplerosis for citric acid cycle function. J Biol Chem. 2002;277(34):30409-12.

\section{Ready to submit your research? Choose BMC and benefit from:}

- fast, convenient online submission

- thorough peer review by experienced researchers in your field

- rapid publication on acceptance

- support for research data, including large and complex data types

- gold Open Access which fosters wider collaboration and increased citations

- maximum visibility for your research: over $100 \mathrm{M}$ website views per year 